\title{
Scalar Field Dark Matter and Galaxy Formation
}

\author{
Miguel Alcubierre ${ }^{1}$, F. Siddhartha Guzmán ${ }^{2}$, Tonatiuh Matos $^{3}$, Darío \\ Núñez ${ }^{1,4}$, L. Arturo Ureña-López ${ }^{5}$, and Petra Wiederhold ${ }^{6}$ \\ 1 Instituto de Ciencias Nucleares, Universidad Autónoma de México, A.P. 70-543, \\ 04510 D.F., México. \\ 2 Albert Einstein Institut, Max Planck Institut für Gravitationsphysik, Am \\ Mühlenberg 1, D-14476 Golm, Germany \\ 3 Departamento de Física, Centro de Investigación y de Estudios Avanzados del IPN, \\ A.P. 14-740, 07000 México D.F., México. \\ 4 Center for Gravitational Physics and Geometry, Penn State University, University \\ Park, PA 16802, USA. \\ 5 Astronomy Centre, University of Sussex, Brighton BN1 9QJ, United Kingdom. \\ ${ }^{6}$ Departamento de Control Automámtico, Centro de Investigación y de Estudios \\ Avanzados del IPN, A.P. 14-740, 07000 México D.F., México.
}

\begin{abstract}
We present a general description of the scalar field dark matter (SFDM) hypothesis in the cosmological context. The scenario of structure formation under such a hypothesis is based on Jeans instabilities of fluctuations of the scalar field. It is shown that it is possible to form stable long lived objects consisting of a wide range of typical galactic masses around $10^{12} M_{\odot}$ once the parameters of the effective theory are fixed with the cosmological constraints. The energy density at the origin of such an object is smooth as it should.
\end{abstract}

\section{Introduction}

It has been established during this conference that the problem of the nature of dark matter is a non trivial puzzle which is missing even candidates that can provide adequate explanations at all relevant scales 国. Here we push forward the simple hypothesis that establishes a real scalar field as a candidate to be the dark matter at both cosmological and galactic scales, and we focus our attention on the possibility that the structures contained in galaxies can be formed under such a hypothesis. The scalar field hypothesis is motivated because the old belief that the whole material content of the Universe is made of quarks, leptons and gauge bosons is now being abandoned due to recent observations and inconsistencies [2]. As it has been suggested, the cure for the CDM models seems to be the addition of a self-interaction between the dust particles, which would be automatically included in the SFDM model just by introducing a potential of self-interaction.

The hypothesis that scalar fields could be the dark matter is not new. For instance, Denhen proposed that the Higgs field could play that role at galactic scale (see [3]), and other authors have suggested that galactic halos are in fact boson stars [it. Maybe the most popular hypothesis concerning scalar fields as 
dark matter is that of the axion stars, which could account for the missing matter as MACHOS at all scales. However, it has been shown that for the possible range of the axion mass such stars should either fragment or be highly unstable in the time-frame of gravitational cooling processes, since the axion tends to form compact objects (oscillatons) in a short dynamical time scale. But, axions become unstable in such a state and they could be discarded as candidates [5]. Nevertheless, when galactic halos are considered as massive objects made of a scalar field in the same spirit as axion stars, it has been shown that a single real scalar field could explain the rotation curves in galaxies [6, ,7]. It was also possible to present a solution that could explain the presence of supermassive compact objects in galactic active nuclei and thus would provide a space-time background for galaxies in terms of scalar fields, which could explain the motion of the visible matter in galaxies [8]. However, one has to take care of the risks of instability pointed out precisely when the axion stars where studied, and thus the range of masses for the scalar field should change dramatically $\left(10^{18}\right.$ times lighter than the Peccei-Quinn axion). Such masses should be restricted by the cosmological scenario.

Once the cosmological and galactic scales have been already motivated, it is now time to show whether or not the parameters fixed with the cosmological observations permit the formation of galaxies assuming that the dark matter is a scalar field.

\section{The Cosmological Model}

Dark energy has been shown to be a fundamental ingredient of any cosmological model. Here we will consider dark energy as a quintessence field consisting of a real scalar field $\Psi$. On the other hand, our dark matter scalar field would be described by a second scalar field $\Phi$. Finally, the ordinary matter of the Universe (baryons, radiation, neutrinos, etc) is represented by a perfect fluid field with density $\rho$ and pressure $p$. We assume the space-time to be FRW, i.e. described by the line element $d s^{2}=-d t^{2}+a(t)^{2}\left(d r^{2}+r^{2} d \Omega^{2}\right)$, thus the equations governing all these matter components read

$$
\begin{aligned}
& H^{2} \equiv\left(\frac{\dot{a}}{a}\right)^{2}=\frac{\kappa_{0}}{3}\left(\rho+\rho_{\Phi}+\rho_{\Psi}\right) \\
& \ddot{\Phi}+3 H \dot{\Phi}+\frac{d V(\Phi)}{d \Phi}=0 \\
& \ddot{\Psi}+3 H \dot{\Psi}+\frac{d \tilde{V}(\Psi)}{d \Psi}=0 \\
& \dot{\rho}+3 H(\rho+p) \quad=0,
\end{aligned}
$$

where $\kappa_{0} \equiv 8 \pi G$. The scalar energy densities (pressures) are $\rho_{\Phi}=\frac{1}{2} \dot{\Phi}^{2}+V(\Phi)$ $\left(p_{\Phi}=\frac{1}{2} \dot{\Phi}^{2}-V(\Phi)\right)$ and $\rho_{\Psi}=\frac{1}{2} \dot{\Psi}^{2}+\tilde{V}(\Psi)\left(p_{\Psi}=\frac{1}{2} \dot{\Psi}^{2}-\tilde{V}(\Psi)\right)$. Here dots denote 
derivative with respect to cosmological time $t$.

The SFDM model 6, 6, 8,9, 10,11,12,13] assumes that both the dark matter and the dark energy are of scalar field nature. A particular model we have considered in the last years is the following: For the dark energy we adopt a quintessence field $\Psi$ with a scalar potential of the form $\tilde{V}(\Psi)=\tilde{V}_{0}\left[\sinh \left(\alpha \sqrt{\kappa_{0}} \Psi\right)\right]^{\beta}$. It has been shown that this quintessence potential is a reliable model for the dark energy, because of its asymptotic behavior [14]. Nevertheless, the main point of the SFDM model is to assume that the dark matter is also a scalar field $\Phi$ endowed with a self-interacting potential (see 11])

$$
V(\Phi)=V_{0}\left[\cosh \left(\lambda \sqrt{\kappa_{0}} \Phi\right)-1\right]
$$

The mass of the scalar field $\Phi$ is defined as $m_{\Phi}^{2}=\left.V^{\prime \prime}\right|_{\Phi=0}=\lambda^{2} \kappa_{0} V_{0}$. This potential allows the scalar field $\Phi$ to behave just as cold dark matter at cosmological scales, provided that the mass of the boson particle is given by

$$
m_{\Phi}^{2}=(1.7 / 3) \lambda^{2}\left(\lambda^{2}-4\right)^{3} \Omega_{0, \gamma}^{-3} \Omega_{0, \mathrm{C} D M}^{4} H_{0}^{2}
$$

where $\left(\Omega_{0, \mathrm{C} D M}, \Omega_{0, \gamma}, H_{0}\right)$ are the current values of the density parameters of cold dark matter and radiation, and the Hubble parameter, respectively. Because of nucleosynthesis constraints[9.15], we have to take $\lambda \geq 5$.

We now analyze the perturbations of the space-time metric due to the presence of the scalar field $\Phi$. First, we consider a linear perturbation given by $h_{i j}$. We work in the synchronous gauge formalism, where the line element is $d s^{2}=a^{2}\left[-d \tau^{2}+\left(\delta_{i j}+h_{i j}\right) d x^{i} d x^{j}\right]$. We must add the perturbed equation for the scalar field $\Phi(\tau) \rightarrow \Phi(\tau)+\phi(k, \tau)$ 15, 16

$$
\ddot{\phi}+2 \mathcal{H} \dot{\phi}+k^{2} \phi+a^{2} V^{\prime \prime} \phi+(1 / 2) \dot{\Phi} \dot{h}=0
$$

to the linearly perturbed Einstein equations in $k$-space $(\boldsymbol{k}=k \hat{k})$. Here, dots are derivatives with respect to the conformal time $\tau$, primes are derivatives with respect to the unperturbed scalar field $\Phi, h$ is the trace of the metric perturbations $h_{i j}$ and $\mathcal{H}$ is the conformal Hubble factor.

It is well known that scalar perturbations can only grow if the $k^{2}$-term in eq. (7) is subdominant with respect to the second derivative of the scalar potential, that is, if $k<a \sqrt{V^{\prime \prime}}$ [17. According to the cosmological solution for potential (5), the scalar wave number defined by $k_{\Phi}=a \sqrt{V^{\prime \prime}}$ has a minimum value given by 12

$$
k_{m i n, \Phi}=1.3 \lambda \sqrt{\lambda^{2}-4} \Omega_{0, \gamma}^{-1 / 2} \Omega_{0, \mathrm{C} D M} H_{0},
$$

It can be assured that there are no scalar perturbations for $k>m_{\Phi}$, that is, bigger than $k_{\Phi}$ today: they must have been completely erased. Besides, modes such that $m_{\Phi}>k>k_{m i n, \Phi}$ must have been damped during certain epochs. 
From this, we conclude that the scalar power spectrum of $\Phi$ will be damped for $k>k_{m i n, \Phi}$ with respect to the standard case. Therefore, the scalar Jeans length associated to potential (5) must be [12]

$$
L_{J}(a)=2 \pi k_{\min , \Phi}^{-1} .
$$

In Fig. 1 we can see the damped density contrast $\delta^{2}$ at a redshift $z=50$ from a complete numerical evolution using CMBFAST. We see that it is possible to suppress the power spectrum in such a manner that it is possible to explain the smooth cores of dark halos in galaxies and the small abundance of dwarf galaxies, as suggested in [18]. The mass power spectrum is related to the CDM case by the semi-analytical relation (see [19])

$$
P_{\Phi}(k) \simeq\left(\frac{\cos x^{3}}{1+x^{8}}\right)^{2} P_{C D M}(k),
$$

but using $x=\left(k / k_{\min , \Phi}\right)$, with $k_{\min , \Phi}$ being the wave number associated to the Jeans length (9). If we take a cut-off of the mass power spectrum at $k=$ $4.5 \mathrm{~h} \mathrm{Mpc}^{-1}$ [18], we can fix the value of the parameter $\lambda$. Using eq. (8) we find that 12]

$$
\begin{aligned}
& \lambda \simeq 20.3 \\
& V_{0} \simeq\left(3.0 \times 10^{-27} M_{P l} \simeq 36.5 \mathrm{eV}\right)^{4} \\
& m_{\Phi} \simeq 9.1 \times 10^{-52} M_{P l} \simeq 1.1 \times 10^{-23} \mathrm{eV}
\end{aligned}
$$

where $M_{P l}=1.22 \times 10^{19} \mathrm{GeV}$ is the Plank mass. All parameters of potential (5) have now been completely determined and we have the right cut-off in the mass power spectrum.

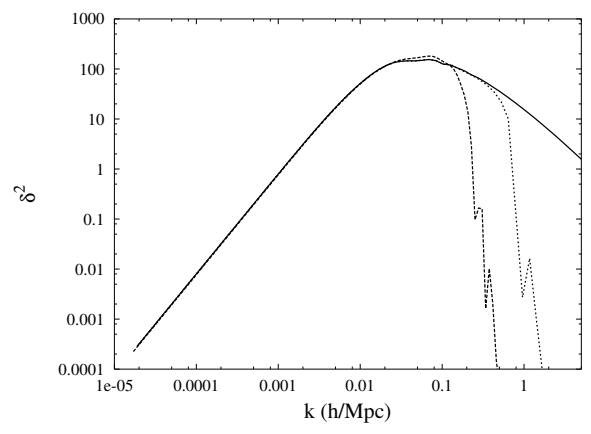

Fig. 1. Power spectrum at a redshift $z=50: \Lambda \mathrm{CDM}$ (solid-curve), and $\Phi$ CDM with $\lambda=5$ (dashed-curve) and $\lambda=10$ (dotted-curve). The normalization is arbitrary. 


\section{Dark Matter in Galaxies}

The problem of galactic dark matter is directly related to the rotation curves of visible matter, for which we started by proposing a model for the dark matter dominated region [6.7]. There, it was assumed that the scalar field was the only contributor to the energy density in that region and thus the one determining the structure of the space-time there; some properties like a relation $p_{r}=10^{6} \rho$ between the radial pressure of the scalar fluid and the energy density of the field were discovered [7]. Nevertheless, it remained unclear if there would be a source that could support such scalar field, since it is well known that there are no nonsingular solutions for spherically symmetric static scalar field configurations. We showed later, through a toy model made of scalar fields, that a ball of dust and an frozen oscillaton could be the source for such a scalar field halo and in one step we found the structure of the space-time from the galactic center to the dark matter dominated region [8]. But probably the major result is the suggested line element found for such a toy model

$$
d s^{2}=-B_{0}\left(r^{2}+b^{2}\right)^{v_{a}^{2}}\left(1-\frac{2 M}{r}\right) d t^{2}+\frac{A_{0}}{\left(1-\frac{2 M}{r}\right)} d r^{2}+r^{2} d \Omega^{2}
$$

with $d \Omega^{2}=d \theta^{2}+\sin ^{2} \theta d \varphi^{2}$ and $M$ a constant related to the mass of the central supermassive object in a way discussed in ref [8]. $B_{0}, b$ and $A_{0}$ are integration constants and $v_{a}$ is the asymptotic value of the velocity of test particles in the sense of the Tully-Fisher relation. This metric is singular at $r=0$, but it has an event horizon at $r=2 M$. This metric does not represent a black hole because it is not asymptotically flat. Nevertheless, for regions where $r \ll b$ but $r>2 M$ the metric behaves like a Schwarzschild black hole. Inside of the horizon the pressure of the perfect fluid is not zero, therefore it does not behave as dust anymore. Thus our toy model is valid only in regions outside the horizon, where it could be an approximation of the galaxy. Metric (12) is not asymptotically flat, but it has a natural cut off when the dark matter density equals the intergalactic density as mentioned in [7].

The charm of such a metric consists in correctly reproducing the rotation curves of galaxies from the center of the galaxy to the dark matter dominated region. The expression for the tangential velocity $v^{\text {rot }}$ of test particles moving along circular stable orbits, as seen by an observed at infinity, is given by $v^{r o t}=$ $\sqrt{r g_{t t, r} /\left(2 g_{t t}\right)}$ for any static spherically symmetric space-time (see $[7]$ ). Thus for (12) this reads

$$
v^{r o t}(r)=\sqrt{\frac{v_{a}^{2}(r-2 M) r^{2}+M\left(r^{2}+b^{2}\right)}{(r-2 M)\left(r^{2}+b^{2}\right)}},
$$

a formula that allows one to fit observational curves. Observe that, for $M=0$, the velocity profile (13) corresponds exactly to the pseudo-isothermal velocity profile for rotation curves [20]. In Figure 2 we show the variation of the rotation 
curve when the value of $M$ changes. It is evident that this would affect only the kinematics in the central parts of the galaxy, exactly in the same way as the mass of the central object should do [21]. In Fig. 3 we present the effect on the rotation curves for test particles traveling on such space-time caused by a change of the parameter $b$ (a sort of core radius). Here one can see different shapes only at large radii, precisely where the dark matter starts to be important. Therefore, considering the visible matter as test particles traveling on such a background space-time the scalar field dark matter in halos seems to be promising.

\section{Collapse of Scalar Field Dark Matter due to Jeans Instabilities}

In order to study the galactic collapse it was necessary to consider a spherically symmetric space-time whose components where time dependent, in such a way that it would be possible to follow the evolution of the system:

$$
d s^{2}=-\alpha^{2}(r, t) d t^{2}+a^{2}(r, t) d r^{2}+r^{2} d \Omega^{2}
$$

using usual spherical coordinates. To simulate a Jeans instability we started by considering the initial scalar field to have a Gaussian distribution $\sqrt{\kappa_{0}} \Phi(x, t=$
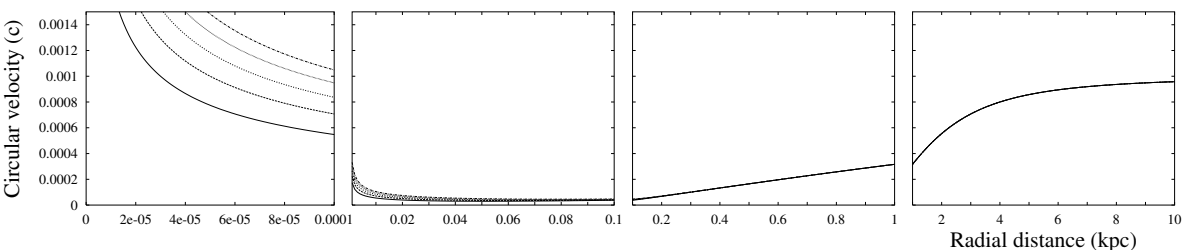

Fig. 2. Rotation curves provided by the line element (10) for different values of the mass of the central object: $M=3,5,7,9,11 \times 10^{6} M_{\odot}$ and fixed $\left(b, v_{a}\right)=\left(3 k p c, 10^{-3} c\right)$ are shown. In the first two plots the rotation curve in the region near the galactic center, where a keplerian fall off can be observed. In the last two plots we show the region where the rotation curve associated to the dark matter component is evident.

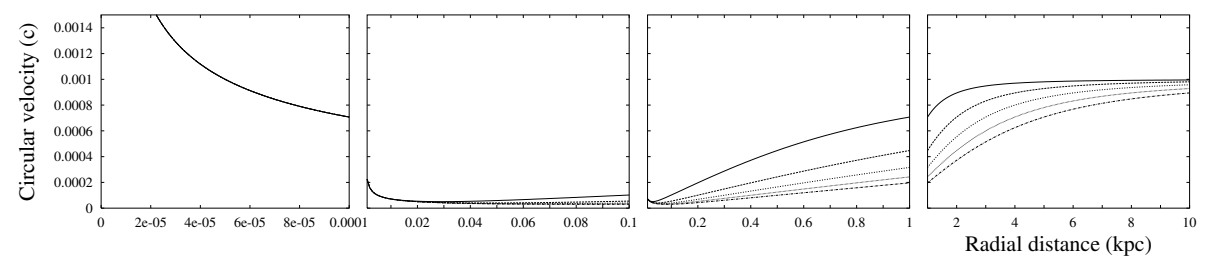

Fig. 3. We show the rotation curves for different values of the parameter $b=$ $1,2,3,4,5 \mathrm{kpc}$ and with $\left(M, v_{a}\right)=\left(5 \times 10^{6} M_{\odot}, 10^{-3} c\right)$ now fixed. It is evident that in such a case the shape of the curve in the region associated with the dark matter changes, but in the center the curves remain unchanged. 
$0)=A e^{-x^{2} / s^{2}}$. The initial mass of the system would change by varying the width and amplitude of such a Gaussian pulse. The energy density is given by

$$
\rho_{s}=\frac{1}{2 a^{2}}\left(\Phi_{, r}^{2}+a^{2} \Phi_{, t}^{2} / \alpha^{2}\right)+V
$$

with $V$ given by (5). According to the results found in [5] the critical mass to have configurations that collapse are found at $M_{\text {crit }} \sim 0.6 m_{P l}^{2} / m \sim 10^{-6} M_{\odot}$ when the mass of the boson is $m \sim 10^{-5} \mathrm{eV}$ (the case of the axion). But in our case the mass of the boson is given by

$$
M_{\text {crit }} \simeq 0.1 \frac{m_{P l}^{2}}{\sqrt{\kappa_{0} V_{0}}}=2.5 \times 10^{13} M_{\odot} .
$$

for the values of the parameters given in (11).

In Fig. 4 we show the total integrated mass of the system, defined as $M(x)=$ $\frac{1}{2} \frac{m_{P l}^{2}}{l} \int_{0}^{x} \rho_{s}(X) X^{2} d X$, as it evolves in time $\left(x=l r, l=\sqrt{\kappa_{0} V_{0}}\right)$. A small drop of $\sim 0.6 \%$ in the total integrated mass can be observed, but numerical convergence tests suggest that most (if not all) of this mass loss is caused by a small amount of numerical dissipation still present in our numerical method. This implies that the system does not radiate any significant amount of energy during the time of the simulation, which indicates that the object is very stable. We also show the

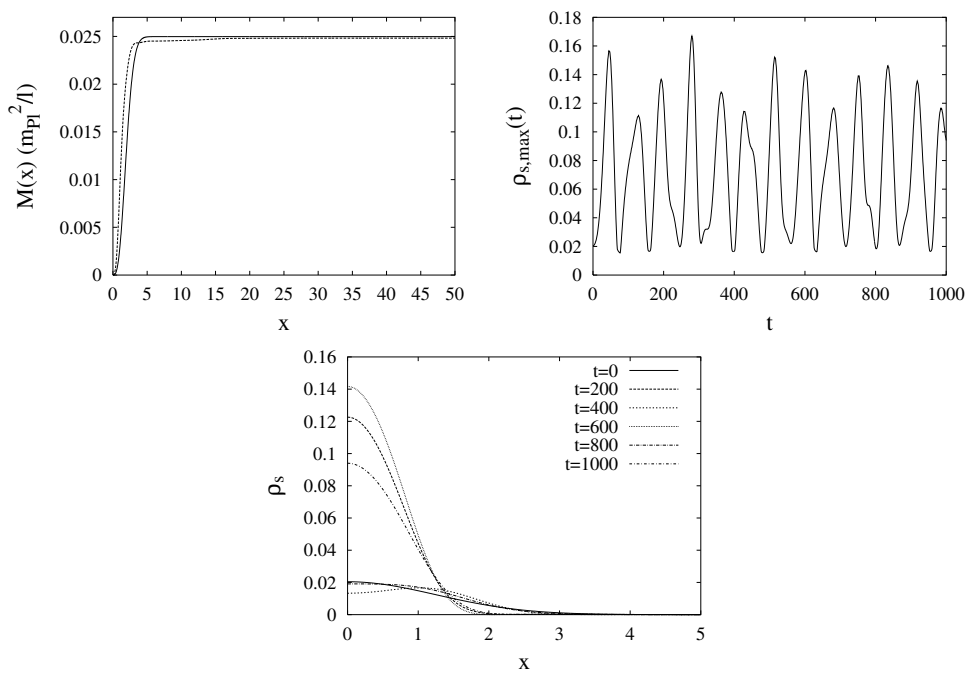

Fig. 4. Left: Integrated mass of the system in terms of time. Right: Temporal evolution of the maximum value of the energy density. The parameters for the initial configuration are $A=0.01, s=2.5$, which correspond to an initial mass of $M_{i}=6.36 \times 10^{12} M_{\odot}$. Center (bottom): Snapshots of the density at different time steps, which shows its smoothness at the origin. 
value of the central density evolving in time. From this plot it is evident that the object is oscillating [22]. Finally, we show the energy density of the scalar field at different times of the evolution. The energy density is clearly smooth at the origin. The boundaries of the object are located at $x=50$ and the evolution was carried out till $t=1000$, which corresponds to some 20 light crossing times through the system.

\section{Concluding remarks}

The importance of the value fixed for $m_{\Phi}$ (5.11), i.e. of the combination of $\lambda$ and $V_{0}$, consists precisely in determining the final stage of the system: if $\lambda$ and $V_{0}$ are much bigger it is easy to show that a system of around $10^{12} M_{\odot}$ would collapse into a black hole, and if they were much smaller the system would simply disperse. Thus, it is important to stress that we have shown that precisely the values fixed by the cosmology are those that provide stable fully relativistic oscillatons with masses around the observed galactic ones.

On the other hand, it could be thought that once the numbers $\lambda$ and $V_{0}$ are fixed, which means that the effective theory coupling the scalar field to gravity has all its parameters fixed, the resulting galaxies should be all of the same mass, but this is not the case. In fact, we have found that there is a window of combinations of the initial parameters $A$ and $s$ that allows the formation of stable objects with different masses. The case presented in Fig. 4 corresponds to a stable and long lived system whose mass is around twice that of the object presented in [23], showing that the galaxies formed through such a process must not have the same mass.

Moreover, the energy density of the collapsed object is smooth, as seen also in Fig. 4, which completes the set of nice properties of the Scalar Field Dark Matter model.

\section{Acknowledgements}

TM would like to thank the organizers for the kind invitation to this conference. This work was partly supported by CONACyT, México under gants 34407-E, 32138-E, 010106 (FSG) and 010385 (LAU). DN acknowledges CONACyT and DGAPA-UNAM grants for partial support. We thank Ed Seidel for

useful discussions. We want to thank Aurelio Espiritu and Erasmo Gomez for thechnical support.

\section{References}

1. J. Ellis, E-print astro-ph/0204059.

2. Shi, X., Schramm, D. N. and Dearborn, D., Phys. Rev. D 50, (1995) 2414. 
3. H. Dehnen and B. Rose, Astrophys. Sp Sci. 207 (1993) 133-144. H. Dehnen, B. Rose and K. Amer, Astrophys. Sp Sci. 234 (1995) 69-83.

4. Franz E. Schunck, E-print astro-ph/9802258.

5. E. Seidel and W. Suen, Phys. Rev. Lett. 72 (1994) 2516.

6. F. S. Guzmán and T. Matos, Class. Quantum Grav. 17 (2000) L9-L16. T. Matos and F. S. Guzmán, Ann. Phys. (Leipzig) 9 (2000) SI 133-136.

7. T. Matos, F. S. Guzmán and D. Núñez, Phys. Rev. D 62 (2000) 061301.

8. T. Matos and F. S. Guzmán, Class. Quantum Grav. 18 (2001) 5055-5064.

9. V. Sahni and L. Wang, Phys. Rev. D 62 (2000) 103517.

10. T. Matos, F. S. Guzmán and L. A. Ureña-López, Class. Quantum Grav. 17 (2000) 1707.

11. T. Matos and L. A. Ureña-López, Class. Quantum Grav. 17 (2000) L71.

12. T. Matos and L. A. Ureña-López, Phys. Rev. D63 (2001) 063506.

13. J. E. Lidsey, T. Matos and L. A. Ureña-López, Phys. Rev. D, in press. E-print astro-ph/0111292.

14. L. A. Ureña-López and T. Matos, Phys. Rev. D 62 (2000) 081302.

15. Pedro G. Ferreira and Michael Joyce, Phys. Rev. D 58 (1998) 023503.

16. R. R. Caldwell, R. Dave and P. J. Steinhardt, Phys. Rev. Lett. 80 (1998) 1582. I. Zlatev, L. Wang and P. J. Steinhardt, Phys. Rev. Lett. 82 (1999) 896. P.J. Steinhardt, L. Wang and I. Zlatev, Phis. Rev. D 59 (1999) 123504.

17. C.-P. Ma et al, Astrophys. J. 521 (1999) L1-L4.

18. Marc Kamionkowski and Andrew R. Liddle, Phys. Rev. Lett. 84 (2000) 4525.

19. W. Hu, R. Barkana and A. Gruzinov, Phys. Rev. Lett. 85, (2000) 1158.

20. W. J. G. de Block, S. S. McGaugh, A. Bosma and V. R. Rubin, ApJ 552 (2001) L23.

21. D. F. Torres, S. Capozziello and G. Lambiase, Phys. Rev. D 62, 104012 (2000).

22. To know more about the numerical methods and the details concerning the simulations see [23].

23. M. Alcubierre, F. S. Guzmán, T. Matos, D. Núñez, L. A. Ureña-López and P. Wiederhold, E-print gr-qc/0110102.

24. L. A. Ureña-López, Class. Quantum Grav., in press. E-print gr-qc/0104093. 